\title{
SURVEY ON TRANSVERSAL NORMED SPACES
}

\author{
Milan R. Tasković*
}

\begin{abstract}
In this paper we formulate a new structure of spaces which we call it transversal (upper or lower) normed spaces. Combination of algebraic and transversal structures opens up the possibility of studying linear transformation of one transversal normed space into another. This concept of spaces is a natural extension of Banach spaces. Most of our work in this paper centers around forms of three fundamental theorems relating to bounded linear transformations: form of the Hahn-Banach theorem, form of the open mapping theorem and form of the Banach

- Steinhaus theorem.
\end{abstract}

\section{Introduction and history}

Starting from the work of Friedrich Riesz and Eduard Helly on the problem of moments it was a natural generalization to define norms on arbitrary vector spaces.

This was done independently by Stefan Banach and Hans Hahn about 1920. In 1932 Banach published book containing a comprehensive account of all results known at that time in the theory of normed spaces, and in particular the theorems he had published in his papers of 1922 and 1929.

A large part was devoted to the concept of weak convergence and its generalizations, which he had begun to study in 1929. Banach's terminology and notations were universally adopted, complete normed spaces became known

AMS (MOS) Subject Classification 1991. Primery: 54E15. Seconadry: 54E35

Key words and phrases: Transversal spaces, transversal normed spaces (upper or lower), upper normed spaces, lower normed spaces, form of the Hahn-Banach theorem, form of the open mapping theorem, form of the Banach-Steinhaus theorem, form of the uniform boundedness theorem.

*Research supported by Science Fund of Serbia under Grant 1457. 
as Banach spaces. After 1935 the theory of normed spaces became part of the more general theory of locally convex spaces.

In connection with this, first, in Tasković [9] we introduced the concept of transversal (upper or lower) spaces as a natural extension of Fréchet's, Kurepa's and Menger's spaces.

Let $X$ be a nonempty set. The function $\rho: X \times X \rightarrow \mathbb{R}_{+}^{0}:=[0,+\infty)$ is called an upper transverse on $X$ (or upper transversal) iff: $\rho[x, y]=\rho[y, x]$, $\rho[x, y]=0$ if and only if $x=y$, and if there is function $\psi:\left(\mathbb{R}_{+}^{0}\right)^{2} \rightarrow \mathbb{R}_{+}^{0}$ such that

$$
\rho[x, y] \leq \max \{\rho[x, z], \rho[z, y], \psi(\rho[x, z], \rho[z, y])\}
$$

for all $x, y, z \in X$. An upper transversal space is a set $X$ together with a given upper transverse on $X$. The function $\psi$ in (A) is called upper bisection function.

On the other hand, the function $\rho: X \times X \rightarrow \mathbb{R}_{+}^{0}$ is called a lower transverse on $X$ (or lower transversal) iff: $\rho[x, y]=\rho[y, x], \rho[x, y]=0$ if and only if $x=y$, and if there is a lower bisection function $d:\left(\mathbb{R}_{+}^{0}\right)^{2} \rightarrow \mathbb{R}_{+}^{0}$ such that

$$
\rho[x, y] \geq \min \{\rho[x, z], \rho[z, y], d(\rho[x, z], \rho[z, y])\}
$$

for all $x, y, z \in X$. A lower transversal space is a set $X$ together with a given lower transverse on $X$.

Also, the function $\rho: X \times X \rightarrow \mathbb{R}_{+}^{0} \cup\{+\infty\}:=W$ is called a lower limit transverse on $X$ iff: $\rho[x, y]=\rho[y, x], \rho[x, y]=+\infty$ if and only if $x=y$, and if there is a lower bisection function $d: W^{2} \rightarrow W$ such that (Am).

A lower limit transversal space is a set $X$ together with a given lower limit transverse on $X$.

For further facts on transversal (upper or lower) spaces see: Tasković [7] and [9].

\section{Transversal upper normed spaces}

Let $X$ be a linear space over $\mathbb{K}(:=\mathbb{R}$ or $\mathbb{C})$. The mapping $x \mapsto\|x\|$ : $X \rightarrow \mathbb{R}$ is called an upper transversal seminorm (or upper seminorm) iff: $\|x\| \geq 0$ for every $x \in X,\|\lambda x\|=|\lambda|\|x\|$ for all $\lambda \in \mathbb{K}$ and $x \in X$, and if there is a function $\psi:\left(\mathbb{R}_{+}^{0}\right)^{2} \rightarrow \mathbb{R}_{+}^{0}:=[0,+\infty)$ such that

$$
\|x+y\| \leq \max \{\|x\|,\|y\|, \psi(\|x\|,\|y\|)\}
$$

for all $x, y \in X$.

Further, $x \mapsto\|x\|$ is called an upper transversal norm (or upper norm) iff in addition: $\|x\|=0$ if and only if $x=0$. 
An upper transversal normed space $(X,\|\cdot\|)$ over $\mathbb{K}$ consists of a linear space $X$ over $\mathbb{K}$ together with an upper transversal norm $x \mapsto\|x\|$.

The function $\psi:\left(\mathbb{R}_{+}^{0}\right)^{2} \rightarrow \mathbb{R}_{+}^{0}$ in $(\mathrm{Nu})$ is called upper bisection function. From $(\mathrm{Nu})$ it follows, by induction, that there is a function $\mathfrak{M}$ : $\left(\mathbb{R}_{+}^{0}\right)^{n} \rightarrow \mathbb{R}_{+}^{0}$ such that

$$
\begin{gathered}
\left\|x_{0}-x_{n}\right\| \leq \\
\leq \max \left\{\left\|x_{0}-x_{1}\right\|, \ldots,\left\|x_{n-1}-x_{n}\right\|, \mathfrak{M}\left(\left\|x_{0}-x_{1}\right\|, \ldots,\left\|x_{n-1}-x_{n}\right\|\right)\right\}
\end{gathered}
$$

for all $x_{0}, x_{1}, \ldots, x_{n} \in X$ and for any fixed integer $n \geq 2$.

It is easy to verify that the upper transversal normed linear space $X$ is a transversal upper space (see: Tasković [7]) with respect to the upper transverse $\rho: X \times X \rightarrow \mathbb{R}_{+}^{0}$ defined by

$$
\rho[x, y]=\|x-y\| \quad \text { for all } \quad x, y \in X
$$

thus we obtain $\rho[x-z, y-z]=\rho[x, y]$ and $\rho[\lambda x, \lambda y]=|\lambda| \rho[x, y]$ for all $x, y, z \in X$ and for every scalar $\lambda \in \mathbb{K}$.

In this sense, an upper transversal normed space $X$ is said to be upper complete if it is upper complete as a transversal upper space. The upper convergence $x_{n} \rightarrow x(n \rightarrow \infty)$ means $\left\|x_{n}-x\right\| \rightarrow 0(n \rightarrow \infty)$.

We will in further denote by $\mathfrak{G}\left(\mathbb{R}_{+}^{0}\right)$ the set of all upper bisection functions $\psi:\left(\mathbb{R}_{+}^{0}\right)^{2} \rightarrow \mathbb{R}_{+}^{0}$ which are increasing satisfying $\psi(t, t) \leq t$ for every $t \in \mathbb{R}_{+}^{0}$.

In an former paper (Tasković: Math. Japonica, 37 (1992), 367-372), have introduced the notion of general convex functions. A function $f: D \rightarrow \mathbb{R}$, where $\mathbb{R}$ denotes the real line and $D$ is a convex subset of $\mathbb{R}^{n}$, is said to be general convex iff there is a function $\psi:(f(D))^{2} \rightarrow \mathbb{R}$ such that

$$
f(\lambda x+(1-\lambda) y) \leq \max \{f(x), f(y), \psi(f(x), f(y))\}
$$

for all $x, y \in D$ and for arbitrary $\lambda \in[0,1]$. We notice that the set of all convex and quasiconvex functions can be a proper subset of the set all general convex functions.

Also, a function $f: D \rightarrow \mathbb{R}$ is said to be general convex with tent iff there is a function $\psi: f(D)^{2} \rightarrow \mathbb{R}$ such that (Max) and that

$$
S:=\left\{(x, y) \in \mathbb{R}^{n+1}: x \in D, y>\max \{f(a), f(b), \psi(f(a), f(b))\}\right\}
$$

is a convex set for arbitrary $a, b \in D$.

We notice that upper transversal norm $x \mapsto\|x\|$ is a general convex function. The proof is simple.

Example 1. Let $X$ be a linear space over $\mathbb{K}$ and let $\mathfrak{G}$ be the set of all nonincreasing, left continuous functions $x \mapsto M_{x}: \mathbb{R} \rightarrow[a, b]$ for $a<b$ 
$\left(a, b \in \mathbb{R}_{+}^{0}\right)$, where $\inf M_{x}=a$ and $\sup M_{x}=b$. Then $(X, \mathfrak{G})$ is an upper transversal normed space if: $M_{x}(0)=a$ for every $x \in X, M_{x}=b$ for $x \leq 0$ and $M_{x}=a$ for $x>0$,

$$
M_{\lambda x}(\sigma)=M_{x}\left(\frac{\sigma}{|\lambda|}\right) \quad \text { for every } \quad x \in X
$$

and for all $\sigma \in \mathbb{R}$ and $\lambda \neq 0$, and if there is a function $g:[a, b]^{2} \rightarrow[a, b]$ such that

$$
M_{x+y}(p+q) \leq \max \left\{M_{x}(p), M_{y}(q), g\left(M_{x}(p), M_{y}(q)\right)\right\}
$$

for all $x, y \in X$ and for all $p, q \geq 0$.

The fundamental results of this section are the statement characterizing finite dimensional spaces (as a Riesz's theorem type), statement of separable upper transversal normed spaces and a statement as the form of Riesz lemma for upper transversal normed spaces. See brief proofs for this in: Tasković [7].

Also, in this part, we derive a general extension theorem, as a form of Hahn-Banach theorem, for linear functionals on an arbitrary linear space.

In the next we can apply this theorem to the problem of the existence of upper bounded linear functionals.

\section{General Hahn-Banach theorem and extension theorems}

In this section we give some generalizations of the well known HahnBanach theorem in terms of general convex functions.

Let $A \subset X$ be a subset of linear space $X$. We say that $A$ is $Q$-radial at a point $a \in A$ if for every $y \in X(y \neq 0)$, there exists an $\varepsilon_{y}>0$ such that $a+\lambda y \in A$ for every $\lambda \in \mathbb{Q} \cap\left(0, \varepsilon_{y}\right)$.

Lemma 1. Let $X$ be a linear space, let $D \subset X$ be a set $Q$-convex and $Q$-radial at a point $x_{0} \in D$, and let $L \subset X$ be a linear space (over $\mathbb{Q}$ ) such that $x_{0} \in L$. Let $f: D \rightarrow \mathbb{R}$ be a function fulfilling the inequality

$$
f(\lambda x+(1-\lambda) y) \leq \max \{f(x), f(y), g(f(x), f(y))\}
$$

for a function $g: f(D)^{2} \rightarrow \mathbb{R}$, for all $x, y \in D$ and for every $\lambda \in \mathbb{Q} \cap[0,1]$. If $z \notin L$, if $Z=\operatorname{Lin}(L \cup\{z\})$ and if $h: L \rightarrow \mathbb{R}$ is a linear functional such that

$$
h(x) \leq f(x) \text { for every } x \in D \cap L,
$$

then there exists a linear functional $H: Z \rightarrow \mathbb{R}$ such that

$$
H(x) \leq f(x) \text { for every } x \in D \cap Z
$$

and $H \mid L=h$, i.e., there is an extension of the linear functional $h$ on $Z$. 
Hence we derive a new form of the rational version of the Hahn-Banach theorem.

Theorem 3.1. Let $X$ be a linear space, let $D \subset X$ be a set $Q$-convex and $Q$-radial at a point $x_{0} \in D$, and let $L \subset X$ be a linear space (over $\mathbb{Q}$ ) such that $x_{0} \in L$. Let $f: D \rightarrow \mathbb{R}$ be a function fulfilling $(Q)$ for all $x, y \in D$ and for every $\lambda \in \mathbb{Q} \cap[0,1]$. If $h: L \rightarrow \mathbb{R}$ is a linear functional with property (1), then there exists a linear functional $H: X \rightarrow \mathbb{R}$ such that

$$
H(x) \leq f(x) \text { for every } x \in D
$$

and $H \mid L=h$, i.e., there is an extension of the linear functional $h$ on $X$.

In this section arguing as in the proof of Theorem 1 we can get however the following result.

Theorem 3.2. (General Hahn-Banach theorem). Let $X$ be a real linear space, let $D \subset X$ be a subspace and let $f: X \rightarrow \mathbb{R}$ be a general convex function such that

$$
h(x) \leq f(x) \text { for every } x \in D,
$$

where $h: D \rightarrow \mathbb{R}$ is a linear functional. Then there is a linear functional $H: X \rightarrow \mathbb{R}$ such that

$$
H(x) \leq f(x) \quad \text { for every } \quad x \in X
$$

and $H \mid D=h$, i.e., there is a linear extension of the linear functional $h$ on the space $X$.

By the preceding method, we also prove the following result, which is a separation theorem on a linear space.

Theorem 3.3. (Separation of concavity and general convexity). Let $E$ be a real linear space, let $D \subset E$ be a nonempty convex subset, and let $f: E \rightarrow \mathbb{R}$ be a general convex with tent function such that

$$
k(x) \leq f(x) \text { for every } x \in D,
$$

where $k: D \rightarrow \mathbb{R}$ is a concave functional. Then there is a linear functional $H: E \rightarrow \mathbb{R}$ such that

$$
k(x) \leq H(x) \quad \text { for every } \quad x \in D,
$$

and

$$
H(y) \leq f(y) \quad \text { for every } y \in E .
$$

A brief proof of this statement may by found in Taskovic [7]. For the proof of this statement the following result is essential. 
Lemma 2. Let $X$ be a nonempty compact convex subset of a separated linear topological space and $f_{v}: X \rightarrow \mathbb{R} \cup\{+\infty\}$ for $v \in I$ a family of lower semi-continuous general convex with tent functionals. If for any finite indices $v_{1}, \ldots, v_{n}$ the following inequality holds

$$
\lambda_{1} f_{v_{1}}(y)+\cdots+\lambda_{n} f_{v_{n}}(y) \leq 0
$$

for every $y \in X$ and for any nonnegative numbers $\lambda_{1}, \ldots, \lambda_{n}$ with property $\lambda_{1}+\cdots+\lambda_{n}=1$, then there is an $x \in X$ such that

$$
f_{v}(x) \leq 0 \quad \text { for every } \quad v \in I .
$$

Let $E$ be a linear functional and let $f: E \rightarrow \mathbb{R}$ be a general convex with tent function. For two nonempty subsets $A$ and $B$ of $E$, we consider a number

$$
f(A, B):=\inf \{f(x-y): x \in A, y \in B\} .
$$

As a directly consequence of the preceding results we obtain the following statement.

Proposition 3.4. Let $E$ be a real linear space and let $f: E \rightarrow \mathbb{R}$ be a general convex with tent function. If $C$ and $D$ are nonempty convex subsets of $E$ such that $f(C, D)>-\infty$, then there is a linear functional $H: E \rightarrow \mathbb{R}$ such that

$$
\inf \{H(x): x \in C\}=f(C, D)+\sup \{H(y): y \in D\}
$$

and

$$
H(x) \leq f(x) \quad \text { for every } \quad x \in E .
$$

Let $X$ be a real linear space, $D$ a convex set in the space $X, k: D \rightarrow \mathbb{R}$ and $f: D \rightarrow \mathbb{R}$ are given functionals, $k_{\beta}: D \rightarrow \mathbb{R}(\beta \in J)$ and $f_{\alpha}: D \rightarrow \mathbb{R}$ $(\alpha \in I)$ denote the sequences of concave and general convex functionals such that $k(x) \leq k_{\beta}(x), f_{\alpha}(x) \leq f(x)$ for all $x \in D$, respectively. Also, let the envelope (of functional $f$ ) $\bar{f}(x)=\sup _{\alpha \in I} f_{\alpha}(x)$ be a general convex with tent function. If the functionals $k: D \rightarrow \mathbb{R}$ and $f: X \rightarrow \mathbb{R}$ have the preceding properties we call that $k$ and $f$ have the envelope majorantes property.

Theorem 3.5. Let $X$ be a real linear space, let $D \subset X$ be a nonempty convex set, and let $k: D \rightarrow \mathbb{R}$ and $f: X \rightarrow \mathbb{R}$ have the envelope majorantes property. Then there is a linear functional $H: X \rightarrow \mathbb{R}$ such that

$$
\begin{gathered}
k(x) \leq H(x) \quad \text { for every } \quad x \in D, \\
H(x) \leq f(x) \quad \text { for every } \quad x \in X
\end{gathered}
$$

if and only if the following inequality holds

$$
\inf _{\beta \in J} K_{\beta}(x) \leq \inf _{\lambda>0} \frac{1}{\lambda} \sup _{\alpha \in I} f_{\alpha}(\lambda x)
$$


for every $x \in \operatorname{Conv} D$, where the functions $K_{\beta}(x)$ are defined by

$$
K_{\beta}(x)=\left\{\begin{array}{lll}
k_{\beta}(x) & \text { for every } \quad x \in D, \\
-\infty & \text { for every } & x \in(\operatorname{Conv} D) \backslash D .
\end{array}\right.
$$

Theorem 3.6. (Extension of Mazur-Orlicz's theorem). Let $X$ be a real linear space, and let $g: X \rightarrow \mathbb{R}$ be a given functional. If $J$ is an arbitrary index set, $\left\{x_{j}: j \in J\right\} \subset X$, and if $\left\{c_{j}: j \in J\right\} \subset \mathbb{R}$, then the system of form

$$
\phi \leq g, \quad c_{j} \leq \phi\left(x_{j}\right) \quad \text { for } \quad j \in J
$$

has a solution $\phi \in X^{*}$ if and only if for every finite set $\{j(1), \ldots, j(n)\} \subset J$ and for every set $\left\{\alpha_{1}, \ldots, \alpha_{n}\right\}$ of nonnegative numbers we have

$$
\sum_{k=1}^{n} \alpha_{k} c_{j(k)} \leq g\left(\sum_{k=1}^{n} \alpha_{k} x_{j(k)}\right) .
$$

If $g: X \rightarrow \mathbb{R}$ is a sublinear functional, then from Theorem 5 we obtain well known result in 1934 of S.Mazur and W.Orlicz.

In this section we will consider a stronger version of the general HahnBanach theorem which will also turn out to be equivalent to the Axiom of Choice.

Theorem 3.7. (An equivalent of Axiom of Choice). Let $M$ be a subspace of real linear space $E$ and $S$ a subset of $E$. Suppose $f: E \rightarrow \mathbb{R}$ is a general convex functional and $h: M \rightarrow \mathbb{R}$ a linear functional such that

$$
h(x) \leq f(x) \quad \text { for every } \quad x \in M .
$$

Then the set $G$ of all $f$-dominated linear extensions of $h$ to $E$ has an element $g: E \rightarrow \mathbb{R}$ such that for all $H \in G$ with $g(s) \leq H(s)$ for all $s \in S$, we have $g(s)=H(s)$ for all $s \in S$. That is, $g$ is $S$-maximal in $G$.

Annotations. We notice that, in special case, if $D \subset \mathbb{R}^{n}$ and if the function $f: D \rightarrow \mathbb{R}$ satisfying the following inequality

$$
f(\lambda x+(1-\lambda) y) \leq \lambda f(x)+(1-\lambda) f(y)
$$

for all $x, y \in D$ and for arbitrary $\lambda \in \mathbb{Q} \cap[0,1]$ the preceding statement of Theorem 1 are considered M E. Kuczma [5] and E. Berz [1].

Also, in the book of Kolmogorov - Fomin [4] there is an extension theorem of Hahn - Banach type of the form Theorem 1 in case when $f: D \rightarrow \mathbb{R}$ (for $D \subset \mathbb{R}^{n}$ ) is a convex function. In connection with this see and: Roberts - Varberg [6]. 
Corollary 3.8. (Form of the Hahn - Banach theorem). Let $X$ be a real linear space, let $D \subset X$ be a subspace and let $x \mapsto\|x\|: X \rightarrow \mathbb{R}$ be an upper transversal norm such that

$$
h(x) \leq\|x\| \text { for every } x \in D,
$$

where $h: D \rightarrow \mathbb{R}$ is a linear functional. Then there is a linear functional $H: X \rightarrow \mathbb{R}$ such that

$$
H(x) \leq\|x\| \quad \text { for every } \quad x \in X
$$

and

$$
H(z)=h(z) \quad \text { for every } \quad z \in D .
$$

Proof. Applying Theorem 2 for the case when $f(x)=\|x\|: X \rightarrow \mathbb{R}$, where $x \mapsto\|x\|$ is an upper transversal norm, directly we obtain this statement.

\section{A sandwich with general convexity}

In is the aim of this section to characterize which can be separated by a general convex function. This leads us to functional inequality for real functions $f, g: I \rightarrow \mathbb{R}(I \subset \mathbb{R}$ is an interval $)$ such that

$$
f(\lambda x+(1-\lambda) y) \leq \max \{g(x), g(y), G(g(x), g(y))\}
$$

for a continuous increasing function $g: g(I)^{2} \rightarrow \mathbb{R}$, for all $x, y \in I$ and for arbitrary $\lambda \in[0,1]$.

Theorem 4.9. Real functions $f, g: I \rightarrow \mathbb{R}(I \subset \mathbb{R}$ is an interval) satisfy $(R)$ for all $x, y \in I$ and arbitrary $\lambda \in[0,1]$ if and only if there exists a general convex function $h: I \rightarrow \mathbb{R}$ for a continuous increasing function $S: h(I)^{2} \rightarrow \mathbb{R}$ such that

$$
f(x) \leq h(x) \leq g(x) \quad \text { for every } \quad x \in I .
$$

Arguing as in the proof of Theorem 7 we can get however the following result.

Theorem 4.10. Real functions $f, g: D \rightarrow \mathbb{R}$ ( $D$ is a convex subset of a vector space) satisfy for a continuous increasing function $G: g(D)^{n} \rightarrow \mathbb{R}$ the following inequality

$$
f\left(\sum_{j=1}^{n} \lambda_{j} x_{j}\right) \leq \max \left\{g\left(x_{1}\right), \ldots, g\left(x_{n}\right), G\left(g\left(x_{1}\right), \ldots, g\left(x_{n}\right)\right)\right\}
$$


for all $n \in \mathbb{N}$, for all $x_{1}, \ldots, x_{n} \in D$ and for reals $\lambda_{1}, \ldots, \lambda_{n} \in[0,1]$ with property $\lambda_{1}+\ldots+\lambda_{n}=1$ if and only if there exists a general convex function $h: D \rightarrow \mathbb{R}$ for a continuous increasing function $S: h(D)^{n} \rightarrow \mathbb{R}$ such that

$$
f(x) \leq h(x) \leq g(x) \text { for every } x \in D .
$$

We notice, if real functions $f$ and $g$, defined on a convex subset $D$ of an $(n-1)$-dimensional real linear space, satisfy (2) if and only if there exists a general convex function $h: D \rightarrow \mathbb{R}$ satisfying (3).

Further applications of Theorem 7 concern solutions of the inequalities for the function $f: J \rightarrow \mathbb{R}\left(J\right.$ either $\mathbb{R}_{+}^{0}$ or $\left.\mathbb{R}\right)$ with

$$
f(\lambda x+(T-\lambda) y) \leq \max \{f(x), f(y), G(f(x), f(y))\}
$$

for a continuous increasing function $g: f(J)^{2} \rightarrow \mathbb{R}$, for all $x, y \in J$, for given $T>0$ and for arbitrary $\lambda \in[0, T]$.

For given $T>0$ and $f: J \rightarrow \mathbb{R}$ we define the function $f_{T}: J \rightarrow \mathbb{R}$ by the formula

$$
f_{T}(x)=T^{-1} f(T x) .
$$

Theorem 4.11. Let $T$ be a positive real number. A function $f: J \rightarrow \mathbb{R}$ satisfies (4) for all $x, y \in J$ and arbitrary $\lambda \in[0, T]$ if and only if there exists a general convex function $\psi: J \rightarrow \mathbb{R}$ for a continuous increasing function $S: \psi(J)^{2} \rightarrow \mathbb{R}$ such that

$$
\psi_{T}(x) \leq f(x) \leq \psi(x) \quad \text { for every } \quad x \in J .
$$

Let $f: I \rightarrow \mathbb{R}(I \subset \mathbb{R}$ is an interval) for a continuous increasing function $G: f(I)^{2} \rightarrow \mathbb{R}$ satisfy the following inequality

$$
f(\lambda x+(T-\lambda) y+(1-T) \xi) \leq \max \{f(x), f(y), G(f(x), f(y))\}
$$

for all $x, y \in I$, for given $T \in(0,1)$, for arbitrary $\lambda \in[0, T]$ and for fixed $\xi \in I$.

Fix a real interval $I$ and a point $\xi \in I$. For $T \in(0,1)$ put

$$
I_{T}^{*}=T I+(1-T) \xi .
$$

Given a real function $\psi$ with the domain containing $I_{T}^{*}$, we define $\psi_{T}^{*}$ : $I \rightarrow \mathbb{R}$ by the formula

$$
\psi_{T}^{*}(x)=T^{-1}(\psi(T x+(1-T) \xi)-(1-T) \psi(\xi)) .
$$

Theorem 4.12. Let $T \in(0,1)$. A function $f: I \rightarrow \mathbb{R}(I \subset \mathbb{R}$ is an interval) satisfies (5) for all $x, y \in I$ and arbitrary $\lambda \in[0, T]$ if and only if there exists a general convex function $\psi: I_{T}^{*} \rightarrow \mathbb{R}$ for a continuous increasing function $S: \psi\left(I_{T}^{*}\right)^{2} \rightarrow \mathbb{R}$ such that

$$
\psi_{T}^{*}(x) \leq f(x) \quad \text { for every } \quad x \in I,
$$


and

$$
f(x) \leq \psi(x) \quad \text { for every } \quad x \in I_{T}^{*} .
$$

Theorem 4.13. (Form of the open mapping theorem). Let $X$ and $Y$ be upper complete upper transversal normed spaces with a bisection function $\psi \in \mathfrak{G}\left(\mathbb{R}_{+}^{0}\right)$. If $A$ is a bounded linear operator of $X$ onto $Y$, then $A$ is an open mapping, i.e., $A(G)$ is an open subset of $Y$ whenever $G$ is an open subset of $X$.

A brief proof of this statement may be found in: Taskovic [7]. The open mapping theorem for complete normed spaces was obtainded by S. Banach in 1929. A general form of the open mapping theorem was obtained by J. Schauder in 1930.

In further, let $B(X, Y)$ denoted the set of all bounded linear transformations of an upper transversal normed space $X$ into an upper transversal normed space $Y$.

Theorem 4.14. (Form of the Banach - Steinhaus theorem). Let $J$ be an index set, $X$ and $Y$ be upper transversal normed spaces with a bisection function $\psi \in \mathfrak{G}\left(\mathbb{R}_{+}^{0}\right), X$ be an upper complete space and $A_{j} \in B(X, Y)$ for every $j \in J$. Then $\sup _{j \in J}\left\|A_{j} x\right\|<\infty$ for every $x \in X$ if and only if the following inequality holds $\sup _{j \in J}\left\|A_{j}\right\|<\infty$.

A brief proof of this theorem may be found in Tasković [7]. The preceding statement, for case of the Banach space $X$ and the normed space $Y$, is the well-known as the uniform boundness principle. This principle first was obtained (for bounded linear functionals) in 1922 by Hahn and in 1923 by Hildebrant. General version of this principle was obtained by Banach and Steinhaus in 1927.

\section{Transversal lower normed spaces}

Let $X$ be a linear space over $\mathbb{K}$. The mapping $x \mapsto\|x\|: X \rightarrow \mathbb{R}$ is called a lower transversal seminorm (or lower seminorm) iff: $\|x\| \geq 0$ for every $x \in X,\|\lambda x\|=|\lambda|\|x\|$ for all $\lambda \in \mathbb{K}$ and $x \in X$, and if there is a function $d:\left(\mathbb{R}_{+}^{0}\right)^{2} \rightarrow \mathbb{R}_{+}^{0}$ such that

$$
\|x+y\| \geq \min \{\|x\|,\|y\|, d(\|x\|,\|y\|)\}
$$

for all $x, y \in X$.

Further, $x \mapsto\|x\|$ is called a lower transversal norm (or lower norm) iff in addition: $\|x\|=0$ if and only if $x=0$; also $x \mapsto\|x\|$ is called a limited lower transversal norm (or limited lower norm) iff in addition: $\|x\|=+\infty$ if and only if $x=0$. 
A lower transversal normed space $(X,\|\cdot\|)$ over $\mathbb{K}$ consists of a linear space $X$ over $\mathbb{K}$ together with a lower transversal norm $x \mapsto\|x\|$.

The function $d:\left(\mathbb{R}_{+}^{0}\right)^{2} \rightarrow \mathbb{R}_{+}^{0}$ in (Nl) is called lower bisection function. From $(\mathrm{Nl})$ it follows, by induction, that there is a function $\mathfrak{D}:\left(\mathbb{R}_{+}^{0}\right)^{n} \rightarrow$ $\mathbb{R}_{+}^{0}$ such that

$$
\begin{gathered}
\left\|x_{0}-x_{n}\right\| \geq \\
\geq \min \left\{\left\|x_{0}-x_{1}\right\|, \ldots,\left\|x_{n-1}-x_{n}\right\|, \mathfrak{D}\left(\left\|x_{0}-x_{1}\right\|, \ldots,\left\|x_{n-1}-x_{n}\right\|\right)\right\}
\end{gathered}
$$

for all $x_{0}, x_{1}, \ldots, x_{n} \in X$ and for any fixed integer $n \geq 2$.

It is easy to verify that the lower transversal normed linear space $X$ is a transversal lower space (see: Tasković [7]) with respect to the lower transverse $\rho: X \times X \rightarrow \mathbb{R}_{+}^{0}$ defined by

$$
\rho[x, y]=\|x-y\| \text { for all } x, y \in X
$$

thus we obtain $\rho[x-z, y-z]=\rho[x, y]$ and $\rho[\lambda x, \lambda y]=|\lambda| \rho[x, y]$ for all $x, y, z \in X$ and for every scalar $\lambda \in \mathbb{K}$.

In this sense, the sequence $\left\{x_{n}\right\}_{n \in \mathbb{N}}$ in $(X,\|\cdot\|)$ converges (or lower converges) to $x \in X$ if the sequence $\left\{x_{n}\right\}_{n \in \mathbb{N}}$ converges (or lower converges) in $(X, \rho)$, i.e., if

$$
\rho\left[x_{n}, x\right]=\left\|x_{n}-x\right\| \rightarrow \infty \quad \text { as } \quad n \rightarrow \infty .
$$

In this sense, a lower transversal normed space $X$ is said to be lower complete (or complete) if it is lower complete as a transversal lower space.

We will, in further, denote by $\mathfrak{D}\left(\mathbb{R}_{+}^{0}\right)$ the set of all lower bisection functions $d:\left(\mathbb{R}_{+}^{0}\right)^{2} \rightarrow \mathbb{R}_{+}^{0}$ which are increasing satisfying $d(t, t) \geq t$ for every $t \in \mathbb{R}_{+}^{0}$.

A function $f: D \rightarrow \mathbb{R}$, where $\mathbb{R}$ denotes the real line and $D$ is a convex subset of $\mathbb{R}^{n}$, is said to be general concave iff there is a function $d:(f(D))^{2} \rightarrow \mathbb{R}$ such that

$$
f(\lambda x+(1-\lambda) y) \geq \min \{f(x), f(y), d(f(x), f(y))\}
$$

for all $x, y \in D$ and for arbitrary $\lambda \in[0,1]$. For this see: Tasković [8].

We notice that lower transversal norm $x \mapsto\|x\|$ is a general concave function. The proof is simple.

Example 2. Let $X$ be a linear space over $\mathbb{K}$ and let $\mathfrak{D}$ be the set of all nondecreasing, leftcontinuous functions $x \mapsto N_{x}: \mathbb{R} \rightarrow[a, b]$ for $a<b$ $\left(a, b \in \mathbb{R}_{+}^{0}\right)$, where $\inf N_{x}=a$ and $\sup N_{x}=b$. Then $(X, \mathfrak{D})$ is a lower transversal normed space if: $N_{x}(0)=b$ for every $x \in X, N_{x}=a$ for $x \leq 0$ and $N_{x}=b$ for $x>0$,

$$
N_{\lambda x}(\sigma)=N_{x}\left(\frac{\sigma}{|\lambda|}\right) \quad \text { for every } \quad x \in X
$$


and for all $\sigma \in \mathbb{R}$ and $\lambda \neq 0$, and if there is a function $d:[a, b]^{2} \rightarrow[a, b]$ such that

$$
N_{x+y}(p+q) \geq \min \left\{N_{x}(p), N_{y}(q), d\left(N_{x}(p), N_{y}(q)\right)\right\}
$$

for all $x, y \in X$ and for all $p, q \geq 0$. For further examples see: Tasković [7].

Example 3. (The space $L B(S)$ ). Let $S$ be a nonempty set and let $L B(S)$ be a set of all lower bounded functions $x: S \rightarrow \mathbb{R}$. Thus we have that $L B(S) \subset \mathbb{R}^{S}$ with the lower transversal norm defined by

$$
\|x\|=\inf \{|x(t)|: t \in S\} ;
$$

and we denote the resulting lower transversal normed space by $L B(S)$. Then $L B(S)$ is a lower complete lower transversal normed space. (A brief poof of this fact may be found in: Tasković [7]).

Lower bounded linear operators. Let $X$ and $Y$ be lower transversal normed spaces over $\mathbb{K}$. The linear operator $A: X \rightarrow Y$ is called lower bounded (or bounded) iff

$$
\inf _{x \in X \backslash\{0\}} \frac{\|A x\|}{\|x\|}>0
$$

and, thus, the set $B(X, Y)$ of lower bounded linear operators from $X$ to $Y$ together with the operator lower norm of the form

$$
\|A\|:=\inf _{x \in X \backslash\{0\}} \frac{\|A x\|}{\|x\|}
$$

is a complete (lower complete) lower transversal normed space over $\mathbb{K}$.

Let $X$ and $Y$ be lower transversal normed spaces, then a map $f: M \subset$ $X \rightarrow Y$ is lower continuous (or continuous) at $x \in M$ iff for every $\varepsilon>0$ there is a $\delta=\delta(\varepsilon)>0$ such that $\|f(x)-f(y)\|>\varepsilon$ whenever $y \in M$ and $\|x-y\|>\delta$.

Proposition 5.15. Let $X$ and $Y$ be lower transversal normed spaces. Then the linear operator $A: X \rightarrow Y$ is lower continuous in $0 \in X$ if and only if it is lower bounded.

A brief proof of this statement may be found in: Tasković [7].

Proposition 5.16. Let $X$ be a lower transversal normed space with the lower bisection function $d \in \mathfrak{D}\left(\mathbb{R}_{+}^{0}\right)$ and let $Y$ be a lower complete lower transversal normed space, then $B(X, Y)$ is a lower complete lower transversal normed space. 
A brief proof of this statement may be found in: Tasković [7].

Series in a lower transversal normed space. Let $X$ be lower transversal normed space. A pair of sequences $\left\{x_{n}\right\}_{n \in \mathbb{N}}$ and $\left\{\sigma_{n}\right\}_{n \in \mathbb{N}}$ is called a series if $\sigma_{n}=x_{0}+x_{1}+\cdots+x_{n}$ for any $n \in \mathbb{N}$. The series is said to lower converge (or converge) to $x \in X$ iff

$$
\left\|\sum_{k=1}^{n} x_{k}-x\right\| \rightarrow \infty \quad(n \rightarrow \infty) ;
$$

and, in this sense, the series $\sum_{k=1}^{\infty}\left\|x_{k}\right\|$ is said to lower converge (or converge) iff

$$
\inf \left\{\left\|x_{n+1}\right\|,\left\|x_{n+2}\right\|, \ldots\right\} \rightarrow \infty \quad(n \rightarrow \infty) .
$$

In connection with this, in a lower transversal normed space $X$, a lower absolutely convergent series $\left\{x_{n}\right\}_{n \in \mathbb{N}}$ is a series such that the series of general term $\left\|x_{n}\right\|$ is lower convergent.

Proposition 5.17. A lower transversal normed space $X$ with the lower bisection function $d \in \mathfrak{D}\left(\mathbb{R}_{+}^{0}\right)$ is lower complete if and only if every lower absolutely convergent series in $X$ is lower convergent in $X$.

A brief proof of this statement may be found in: Tasković [7].

The set $M$ is lower dense (or dense) in the lower transversal normed space $X$ iff $C l(M)=X$, i.e., for every $x \in X$ there exists a sequence $\left\{x_{n}\right\}_{n \in \mathbb{N}}$ in $M$ such that $x_{n} \rightarrow x$ as $n \rightarrow \infty$. This is equivalent to the condition that for every $x \in X$ and every $\varepsilon>0$ there is a point $y \in M$ such that $\|x-y\|>\varepsilon$.

A lower transversal normed space $X$ is separable (or lower separable) iff it contains a lower dense set $M$ which is at most countable.

Example 4. Let $K$ be a nonempty compact set in $\mathbb{R}^{n}$ and let $C(K)$ denote the set of all lower continuous functions $f: K \rightarrow \mathbb{R}$. The lower transversal norm

$$
\|f\|=\min _{x \in K}|f(x)|
$$

makes $C(K)$ into a real complete lower transversal normed space. Then the set $M$ of all polynomials in $C(K)$ is lower dense in $C(K)$. The space $C(K)$ is lower separable, for example.

A set $F$ of the lower transversal normed space $X$ is called lower fundamental (or fundamental) iff the linear hull of $F$ is a lower dense set in $X$; if $F$ in addition and countable, then $F$ is called lower fundamental (or fundamental) sequence in $X$. 
Proposition 5.18. The lower transversal normed space $X$ with the lower bisection function $d \in \mathfrak{D}\left(\mathbb{R}_{+}^{0}\right)$ is separable if and only if there is a lower fundamental sequence in $X$.

We notice, from the preceding facts, that a nonempty bounded closed subset of a finite dimensional lower transversal normed linear space is compact. There is a converse of this fact which is true and which provides an important characterization of finite dimensional lower transversal normed linear spaces. We shall need an essential statement.

Proposition 5.19. (Geometrical lemma). Let $X$ be a lower transversal normed space and let $Y$ be a linear subspace of $X$ that is a proper closed subset of $X$. Then for every $\varepsilon>1$ there exists a point $x_{\varepsilon} \in X$ such that

$$
\left\|x_{\varepsilon}\right\|=1 \quad \text { and } \operatorname{hdiam}\left(x_{\varepsilon}, Y\right):=\sup _{y \in Y}\left\|x_{\varepsilon}-y\right\|<\varepsilon \text {. }
$$

In further, applying Geometrical lemma, we obtain the following statement for lower transversal normed spaces.

Proposition 5.20. Let $X$ be a lower transversal normed space and let $d(K[0,1]):=\{x \in X:\|x\| \geq 1\}$ be a closed compact unit ball in $X$, then $X$ is a finite dimensional space.

We turn now to the question of the existence of nonzero lower bounded linear functionals on an arbitrary nonzero lower transversal normed space. Such functionals obviously exist when the space is onedimensional and every nonzero linear space has onedimensional linear subspaces. In this section we derive a general extension statement.

Theorem 5.21. Let $X$ be a real linear space, let $D \subset X$ be a subspace and let $x \mapsto\|x\|: X \rightarrow \mathbb{R}$ be a lower transversal norm such that

$$
h(x) \geq\|x\| \text { for every } x \in D,
$$

where $h: D \rightarrow \mathbb{R}$ is a linear functional. Then there is a linear functional $H: X \rightarrow \mathbb{R}$ such that

$$
H(x) \geq\|x\| \quad \text { for every } \quad x \in X
$$

and

$$
H(z)=h(z) \quad \text { for every } \quad z \in D .
$$

Lemma 3. Let $X$ be a linear space, let $D \subset X$ be a set $Q$-convex and $Q$-radial at a point $x_{0} \in D$, and let $L \subset X$ be a linear space (over $\mathbb{Q}$ ) such that $x_{0} \in L$. Let $f: D \rightarrow \mathbb{R}$ be a function fulfilling the inequality

$$
f(\lambda x+(1-\lambda) y) \geq \min \{f(x), f(y), d(f(x), f(y))\}
$$


for a function $d: f(D)^{2} \rightarrow \mathbb{R}$, for all $x, y \in D$ and for every $\lambda \in \mathbb{Q} \cap[0,1]$. If $z \notin L$, if $Z=\operatorname{Lin}(L \cup\{z\})$ and if $h: L \rightarrow \mathbb{R}$ is a linear functional such that

$$
h(x) \geq f(x) \text { for every } x \in D \cap L,
$$

then there exists a linear functional $H: Z \rightarrow \mathbb{R}$ such that

$$
H(x) \geq f(x) \text { for every } x \in D \cap Z
$$

and $H \mid L=h$, i.e., there is an extension of the linear functional $h$ on $Z$.

Hence we derive a totally new form of the rational version of the well known Hahn-Banach theorem.

Theorem 5.22. Let $X$ be a linear space, let $D \subset X$ be a set $Q$-convex and $Q$-radial at a point $x_{0} \in D$, and let $L \subset X$ be a linear space (over $\mathbb{Q}$ ) such that $x_{0} \in L$. Let $f: D \rightarrow \mathbb{R}$ be a function fulfilling (M) for all $x, y \in D$ and for every $\lambda \in \mathbb{Q} \cap[0,1]$. If $h: L \rightarrow \mathbb{R}$ is a linear functional with property (6), then there exists a linear functional $H: X \rightarrow \mathbb{R}$ such that

$$
H(x) \geq f(x) \text { for every } x \in D
$$

and $H \mid L=h$, i.e., there is an extension of the linear functional $h$ on $X$.

In this section arguing as in the proof of Theorem 14 we can get however the following result.

Theorem 5.23. (Form of Hahn-Banach theorem). Let $X$ be a real linear space, let $D \subset X$ be a subspace and let $f: X \rightarrow \mathbb{R}$ be a general concave function such that

$$
h(x) \geq f(x) \quad \text { for every } \quad x \in D,
$$

where $h: D \rightarrow \mathbb{R}$ is a linear functional. Then there is a linear functional $H: X \rightarrow \mathbb{R}$ such that

$$
H(x) \geq f(x) \quad \text { for every } \quad x \in X
$$

and $H \mid D=h$, i.e., there is a linear extension of the linear functional $h$ on the space $X$.

A brief proof of Theorem 15 (as and Lemma 3 and Theorem 14) may be found in Tasković [7].

Proof of Theorem 13. Applying Theorem 15 for the case when $f(x)=$ $\|x\|: X \rightarrow \mathbb{R}$, where $x \mapsto\|x\|$ is a lower transversal norm, directly we obtain this statement.

We notice that Theorem 15 guarantees that a lower transversal normed space is richly supplied with continuous linear functionals, and makes possible an adequate theory of conjugate spaces.

The following form of open mapping theorem enables us to give a satisfactory description of the projections on a lower transversal normed spase, an has the important closed graph statement as one of its consequences. 
Theorem 5.24. (Form of the open mapping theorem). Let $X$ and $Y$ be lower complete lower transversal normed space with a bisection function $d \in \mathfrak{D}\left(\mathbb{R}_{+}^{0}\right)$. If $A$ is a lower bounded linear operator of $X$ onto $Y$, then $A$ is an open mapping, i.e., $A(G)$ is an open subset of $Y$ whenever $G$ is an open subset of $X$.

A brief proof of this statement may be found in: Tasković [7].

Theorem 5.25. (Form of Banach - Steinhaus theorem). Let $J$ be an index set, $X$ and $Y$ be lower transversal normed spaces with coninuous lower norm and with a bisection function $d \in \mathfrak{D}\left(\mathbb{R}_{+}^{0}\right), X$ be a lower complete space and $A_{j} \in B(X, Y)$ for every $j \in J$. Then $\inf _{j \in J}\left\|A_{j} x\right\|>0$ for every $x \in X$ if and only if $\inf _{j \in J}\left\|A_{j}\right\|>0$.

Proof. First, let $J$ be a countable set and defined for $k \in \mathbb{N}$ the following set such that

$$
E_{k}=\left\{x \in X:\left\|A_{n} x\right\| \geq k \text { for every } n \in \mathbb{N}\right\} .
$$

We shall show that $E_{k}$ (for $k \in \mathbb{N}$ ) is closed. If $x \in C l\left(E_{k}\right)$, then there is a sequence $\left\{x_{i}\right\}_{i \in \mathbb{N}}$ in $E_{k}$ such that $x_{i} \rightarrow x(i \rightarrow \infty)$. Thus we obtain, since $A_{n} \in B(X, Y)$, the following inequality $\left\|A_{n} x\right\| \geq k$, which means that $x \in E_{k}$, i.e., $E_{k}$ is a closed subset in $X$.

Since $X=\cup_{k=1}^{\infty} E_{k}$, applying Baire's theorem for lower transversal space (see: Tasković [7]), we have that there is $k(0) \in \mathbb{N}$ such that $E_{k(0)}$ is a not dense set. Since the set $E_{k(0)}$ is a closed subset of $X$, hence there exists an open ball $K_{0} \equiv d\left(K\left(x_{0}, r\right)\right) \subset E_{k(0)}$.

Let $x \in X(x \neq 0)$ and let $z=x_{0}+\lambda x$, where $\lambda=2 r /\left\|x_{0}\right\|$. From $\left\|z-x_{0}\right\|=\lambda\|x\|=2 r$ we have $z \in K_{0}$. Also, $x_{0} \in K_{0}$ and $K_{0} \subset E_{k(0)}$ implies that

$$
\left\|A_{n} z\right\| \geq k(0) \quad \text { and } \quad\left\|A_{n} x_{0}\right\| \geq k(0) \quad \text { for } \quad n \in \mathbb{N} ;
$$

and hence, since $X$ and $Y$ are lower transversal normed space, we obtain the following inequalities

$$
\begin{gathered}
\left\|A_{n} x\right\|=\frac{1}{\lambda}\left\|A_{n}\left(z-x_{0}\right)\right\| \geq \\
\geq \frac{1}{\lambda} \min \left\{\left\|A_{n} z\right\|,\left\|A_{n} x_{0}\right\|, d\left(\left\|A_{n} z\right\|,\left\|A_{n} x_{0}\right\|\right)\right\} \geq \\
\geq \frac{1}{\lambda} \min \left\{\left\|A_{n} z\right\|,\left\|A_{n} x_{0}\right\|, d\left(\min \left\{\left\|A_{n} z\right\|,\left\|A_{n} x_{0}\right\|\right\}, \min \left\{\left\|A_{n} z\right\|,\left\|A_{n} x_{0}\right\|\right\}\right)\right\} \geq \\
\geq \frac{1}{\lambda} \min \left\{\left\|A_{n} z\right\|,\left\|A_{n} x_{0}\right\|, \min \left\{\left\|A_{n} z\right\|,\left\|A_{n} x_{0}\right\|\right\}\right\} \geq
\end{gathered}
$$




$$
\begin{gathered}
\geq \frac{1}{\lambda}\{k(0), k(0), \min \{k(0), k(0)\}\}= \\
=\frac{k(0)}{\lambda}=\frac{k(0)}{2 r}\|x\| .
\end{gathered}
$$

Thus we obtain $\left\|A_{n}\right\| \geq k(0) / 2 r$ for every $n \in \mathbb{N}$, i.e., the following fact holds

$$
\inf _{n \in \mathbb{N}}\left\|A_{n}\right\| \geq \frac{k(0)}{2 r}>0,
$$

in the case $\operatorname{card}(J)=\aleph_{0}$. If $\operatorname{card}(J)>\aleph_{0}$ and $\inf _{j \in J}\left\|A_{j}\right\|=0$, then there exists an operator sequence $A_{n}(n \in \mathbb{N})$ such that $\lim _{n \rightarrow \infty}\left\|A_{n}\right\|=0$. But, it is in contradiction with (7). Conversely is immediate since $\left\|A_{j} x\right\| \geq\left\|A_{j}\right\|\|x\|$. The proof is complete.

Theorem 5.26. (Form of convergence principle). Let $X$ and $Y$ are lower transversal normed spaces with continuous lower norm and with a bisection function $d \in \mathfrak{D}\left(\mathbb{R}_{+}^{0}\right), Y$ is a lower complete space and $\left\{A_{n}\right\}_{n \in \mathbb{N}}$ the sequence of lower bounded linear operators of $X$ into $Y$ such that:

1) $\left\|A_{n}\right\| \geq \mu$ for every $n \in \mathbb{N}$;

2) There exists $\lim _{n \rightarrow \infty} A_{n} x$ for all points $x \in D$ in a dense subset $D$ of a boll $K:=d\left(K\left(x_{0}, r\right)\right)$ in $X$.

A brief proof of this statement may be found in: Tasković [7].

Lower total continuous operators. Let $X$ and $Y$ are lower transversal normed spaces. The linear operator $A: X \rightarrow Y$ is called lower total continuous iff every lower bounded set in $X$ maps in a relatively compact set in $Y$.

Proposition 5.27. Let $X$ and $Y$ are lower complete lower transversal normed spaces with the bisection function $d \in \mathfrak{D}\left(\mathbb{R}_{+}^{0}\right)$ and let $A_{n}: X \rightarrow Y$ $(n=1,2, \ldots)$ be a sequence of lower total coninuous operators. If

$$
\left\|A_{n}-A\right\| \rightarrow \infty \quad(\text { as } n \rightarrow \infty),
$$

i.e., if the sequence of operators $\left\{A_{n}\right\}_{n \in \mathbb{N}}$ lower uniformly converges to $A$, then $A$ is a lower total coninuous operator.

In further, we notice that with the help of Geometrical lemma (Proposition 6) we now obtain the following statement.

Proposition 5.28. (Form of Fredholm alternative). Let $X$ be a lower complete lower transversal normed space and let $A: X \rightarrow X$ be a linear lower total continuous operator. Then, or the equation

$$
x-A x=y
$$

has a unique solution for every $y \in X$, or the equation $x-A x=0$ has a nontrivial solution. 
A brief proof of this statement may be found in: Tasković [7].

Lower summability. In this section we shall consider an application of the form of principle of uniform boundedness (Theorems 16 and 17) in classical analysis. In this sense let $A=\left(\alpha_{m n}\right)$ be a double sequence of real numbers. A sequence $\left(\zeta_{n}\right)$ of real numbers is said to be lower $A$-summable to the limit $\zeta$ iff the series

$$
\eta_{i}=\sum_{k=1}^{\infty}\left|\alpha_{i k}\right| \zeta_{k}
$$

lower converges for each positive integer $i$ and if the sequence $\left(\eta_{i}\right)$ is lower converges to $\zeta$ as $i \rightarrow \infty$. In this case denote that $\zeta=l\left(A-\lim _{i \rightarrow \infty} \eta_{i}\right)$.

A double sequence $A=\left(\alpha_{m n}\right)$ is said to determine a lower regular method of summability iff each lower convergent sequence $\left(\zeta_{n}\right)$ of real numbers is lower $A$-summable to $\lim _{n \rightarrow \infty} \zeta_{n}$.

Proposition 5.29. (Characterization of lower $A$-summable). A double sequence $A=\left(\alpha_{m n}\right)$ determines a lower regular method of summability if and only if:

(a) $\lim _{i \rightarrow \infty} \sum_{k=1}^{\infty}\left|\alpha_{i k}\right|=1$

(b) $\left\{\sum_{k=1}^{\infty}\left|\alpha_{i k}\right|^{q}\right\}^{1 / q} \geq \mu>0$ for every $i \in \mathbb{N}$, for $0<p<1$ and $1 / p+1 / q=1$.

This result is connected, de facto, with the classical result on regular method of summability which is due to Toeplitz, see: Brown - Page [2, p. 315]. A brief proof of Proposition 10 may be found in: Tasković [7].

General annonation. For further applications of the preceding methods of this paper see: Tasković [7].

\section{Further facts}

Let $X$ be a linear space over $\mathbb{K}$. The mapping $x \mapsto\|x\|: X \rightarrow \mathbb{R}$ is called an upper global transversal seminorm (or upper global seminorm) iff: $\|x\| \geq 0$ for every $x \in X,\|\lambda x\|=|f(\lambda)|\|x\|$ for all $\lambda \in \mathbb{K}$ and $x \in X$ where $f: \mathbb{K} \rightarrow \mathbb{K}$, and if there is a function $\psi:\left(\mathbb{R}_{+}^{0}\right)^{2} \rightarrow \mathbb{R}_{+}^{0}$ such that $(\mathrm{Nu})$.

Further, $x \mapsto\|x\|$ is called an upper global transversal norm (or upper global norm) iff in addition: $\|x\|=0$ if and only if $x=0$. An upper global transversal normed space or upper global normed space $(X,\|\cdot\|)$ over $\mathbb{K}$ consists of a linear space $X$ over $\mathbb{K}$ together with an upper global transversal norm $x \mapsto\|x\|$.

The mapping $x \mapsto\|x\|: X \rightarrow \mathbb{R}$ is called a lower global transversal seminorm (or lower global seminorm) iff: $\|x\| \geq 0$ for every $x \in X$, 
$\|\lambda x\|=|f(\lambda)|\|x\|$ for all $\lambda \in \mathbb{K}$ and $x \in X$ where $f: \mathbb{K} \rightarrow \mathbb{K}$, and if there is a function $d:\left(\mathbb{R}_{+}^{0}\right)^{2} \rightarrow \mathbb{R}_{+}^{0}$ such that $(\mathrm{Nl})$.

Further, $x \mapsto\|x\|$ is called a lower global transversal norm (or lower global norm) iff in addition: $\|x\|=0$ if and only if $x=0$; also, $x \mapsto\|x\|$ is called a limited lower global transversal norm (or limited lower global norm) iff in addition: $\|x\|=+\infty$ if and only if $x=0$.

A lower global transversal normed space or lower global normed space $(X,\|\cdot\|)$ over $\mathbb{K}$ consists of a linear space $X$ over $\mathbb{K}$ together with a lower global transversal norm $x \mapsto\|x\|$.

A limited lower global transversal normed space or limited lower global normed space $(X,\|\cdot\|)$ over $\mathbb{K}$ consists of a linear space $X$ over $\mathbb{K}$ together with a limited lower global transversal norm $x \mapsto\|x\|$.

We notice that every upper transversal normed space $(X,\|\cdot\|)$ is a limited lower global transversal normed space of the form $(X, 1 /\|\cdot\|)$.

Example 5. (The space $l_{p}^{n}$ for $1 \leq p<\infty$ ). Let $p$ be a real number such that $1 \leq p<\infty$. We denote by $l_{p}^{n}$ the space of all $n$-tuples $x=\left(x_{1}, x_{2}, \ldots, x_{n}\right)$ of scalars, with the limited lower global transversal norm defined by

$$
\|x\|_{p}=\frac{1}{\left(\sum_{k=1}^{n}\left|x_{k}\right|^{p}\right)^{1 / p}} .
$$

Example 6. (The space $l_{p}=l_{p}^{\infty}$ for $1 \leq p<\infty$ ). We again consider a real number $p$ with property that $1 \leq p<\infty$, and we denote by $l_{p}$ the space of all sequences $x=\left(x_{1}, x_{2}, \ldots\right)$ of scalars such that $\sum_{k=1}^{\infty}\left|x_{k}\right|^{p}<\infty$, with the limited lower global transversal norm defined by

$$
\|x\|_{p}=\frac{1}{\left(\sum_{k=1}^{\infty}\left|x_{k}\right|^{p}\right)^{1 / p}} .
$$

Example 7. (The space $L_{p}$ for $1 \leq p<\infty$ ). An $L_{p}$ space essentially consists of all measurable functions $f$ defined on a measure space $X$ with measure $m$ which are such that $|f(x)|^{p}$ is integrable, with

$$
\|f\|_{p}=\frac{1}{\left(\int|f(x)|^{p} d m(x)\right)^{1 / p}}
$$

taken as the limited lower global transversal norm.

Since integration is a generalized type of summation, formulas (8) and (9) are special cases of (10). 
Example 8. (Transversal locally convex spaces). An upper transversal locally convex space $\left(X,\left\{p_{j}\right\}_{j \in J}\right)$ consists of a linear space $X$ over $\mathbb{K}$ together with a system of upper transversal seminorms $\left\{p_{j}\right\}_{j \in J}$ such that

$$
p_{j}(x)=0 \quad \text { if and only if } \quad x=0
$$

for all $j \in J$. The set $U$ in $X$ is called upper open iff for every $x_{0} \in U$ there is an $\varepsilon>0$ and there are finitely many indices $j_{1}, \ldots, j_{n} \in J$ such that the set

$$
\left\{x \in X: p_{j(k)}\left(x-x_{0}\right)<\varepsilon, \quad k=1, \ldots, n\right\}
$$

is contained in $U$. These upper open sets form a topology $\tau$, which makes $X$ into a separated (Hausdorff) tpological space.

A lower transversal locally convex space $\left(X,\left\{p_{j}\right\}_{j \in J}\right)$ consists of a linear space $X$ over $\mathbb{K}$ together with a system of lower transversal seminorms $\left\{p_{j}\right\}_{j \in J}$ such that

$$
p_{j}(x)=0 \quad\left(\text { or } p_{j}(x)=+\infty\right) \quad \text { if and only if } \quad x=0
$$

for all $j \in J$. The set $U$ in $X$ is called lower open iff for every $x_{0} \in U$ there is an $\varepsilon>0$ and there are finitely many indices $j_{1}, \ldots, j_{n} \in J$ such that the set

$$
\left\{x \in X: p_{j(k)}\left(x-x_{0}\right)>\varepsilon, \quad k=1, \ldots, n\right\}
$$

is contained in $U$. These lower open sets form a topology $\tau$, which makes $X$ into a separated (Hausdorff) topological space.

Example 9. (Edges normed spaces). Let $X$ be a linear space over $\mathbb{K}$. The mapping $x \mapsto\|x\|: X \rightarrow[a, b]$ for $a<b\left(a, b \in \mathbb{R}_{+}^{0}\right)$ is called an upper edges seminorm iff: $\|\lambda x||=|\lambda|\| x \|$ for all $\lambda \in \mathbb{K}$ and $x \in X$, and if there is a function $\psi:[a, b]^{2} \rightarrow[a, b]$ such that $(\mathrm{Nu})$.

Further, $x \mapsto\|x\|$ is called an upper edges norm iff in addition: $\|x\|=a$ if and only if $x=0$. An upper edges normed space $(X,\|\cdot\|)$ over $\mathbb{K}$ consists of a linear space $X$ over $\mathbb{K}$ together with an upper edges norm $x \mapsto\|x\|$.

The upper edges convergence $x_{n} \rightarrow x(n \rightarrow \infty)$ means that $\rho\left[x_{n}, x\right]=$ $\left\|x_{n}-x\right\| \rightarrow a(n \rightarrow \infty)$. The former example 1 is a characteristic example of an upper edges normed space.

The mapping $x \mapsto\|x\|: X \rightarrow[a, b]$ for $a<b\left(a, b \in \mathbb{R}_{+}^{0}\right)$ is called a lower edges seminorm iff: $\|\lambda x\|=|\lambda|\|x\|$ for all $\lambda \in \mathbb{K}$ and $x \in X$, and if there is a function $d:[a, b]^{2} \rightarrow[a, b]$ such that $(\mathrm{Nl})$.

Further, $x \mapsto\|x\|$ is called a lower edges norm iff in addition: $\|x\|=b$ if and only if $x=0$. A lower edges normed space $(X,\|\cdot\|)$ over $\mathbb{K}$ consists of a linear space $X$ over $\mathbb{K}$ together with a lower edges norm $x \mapsto\|x\|$. 
The lower edges convergence $x_{n} \rightarrow x(n \rightarrow \infty)$ means that $\rho\left[x_{n}, x\right]=$ $\left\|x_{n}-x\right\| \rightarrow b(n \rightarrow \infty)$. The former example 2 is a characteristic example of a lower edges normed space.

Example 10. (Transversal semi-inner spaces). A complex (real) vector space $X$ over $\mathbb{K}$ is called transversal upper semi-inner space if corresponding to any pair of elements $x, y \in X$, there is defined a complex (real) number $[x, y]$ which satisfies the following properties: $[x, x]>0$ for $x \neq 0$, $[\lambda x, y]=\lambda[x, y]$ for all $x, y \in X$ and $\lambda \in \mathbb{K},[x+y, z]=[x, z]+[y, z]$ for all $x, y, z \in X$, and if there is a function $\psi: \mathbb{K}^{2} \rightarrow \mathbb{K}$ such that

$$
|[x, y]|^{2} \leq \max \{[x, x],[y, y], \psi([x, x],[y, y])\}
$$

for all $x, y \in X$. We put $\|x\|=[x, x]^{1 / 2}$ and thus $X$ is an upper transversal normed space.

A complex (real) vektor space $X$ over $\mathbb{K}$ is called transversal lower semi-inner space if corresponding to any pair of elements $x, y \in X$, there is defined a complex real number $[x, y]$ which satisfies the following properties: $[x, x]>0$ for $x \neq 0,[\lambda x, y]=\lambda[x, y]$ for all $x, y \in X$ and $\lambda \in \mathbb{K}$, $[x+y, z]=[x, z]+[y, z]$ for all $x, y, z \in X$ and if there is a function $d: \mathbb{K}^{2} \rightarrow \mathbb{K}$ such that

$$
|[x, y]|^{2} \geq \min \{[x, x],[y, y], d([x, x],[y, y])\}
$$

for all $x, y \in X$. We put $\|x\|=[x, x]^{1 / 2}$ and thus $X$ is a lower transversal normed space.

For further facts on transversal semi-inner spaces see: Tasković [7].

Example 11. (Transversal normed algebras). An upper transversal normed algebra $X$ is a complex upper transversal normed space which is also an algebra with identity 1 , and in which the multiplicative structure is related to the upper norm by the following requirements: $\|1\|=1$ and there is a function $g:\left(\mathbb{R}_{+}^{0}\right)^{2} \rightarrow \mathbb{R}_{+}^{0}$ such that

$$
\|x y\| \leq \max \{\|x\|,\|y\|, g(\|x\|,\|y\|)\}
$$

for all $x, y \in X$. An upper transversal normed subalgebra of $X$ is a closed subalgebra of $X$ which contains 1 .

A lower transversal normed algebra $X$ is a complex lower transversal normed space which is also an algebra with identity 1 , and in which the multiplictive structure is related to the lower norm, by the following requirements: $\|1\|=1$ and there is a function $h:\left(\mathbb{R}_{+}^{0}\right)^{2} \rightarrow \mathbb{R}_{+}^{0}$ such that

$$
\|x y\| \geq \min \{\|x\|,\|y\|, h(\|x\|,\|y\|)\}
$$

for all $x, y \in X$. A lower transversal normed subalgebra of $X$ is a closed subalgebra of $X$ which contains 1. (For further facts see: Tasković [7]). 


\section{References}

[1] E. Berz: Sublinear functions on $\mathbb{R}$, Aequationes Math., 12 (1975), 200-206.

[2] A. L. Brown, and A. Page: Elements of Functional Analysis, Van Nostrand Reinhold Company, London, First published 1970, 394 pages.

[3] N. Dunford, and J. T. Schwartz: Linear Operators, Part I: General theory, Interscience Publishers Ltd., London and Inc., New York, 1958, 858 pages.

[4] A. N. Kolmogorov and S. V. Fomin (А. Н. Колмогоров анд С. В. Фомин): Elementi teoriji funkcij i funkcionalnogo analiza (Элементы теории функций и функционального анализа), Izdatelstvo Nauka, Moskva 1973. (First edition: Izdatelstvo Nauka, Moskva 1968).

[5] M. Kuczma: Note on convex functions, Ann. Univ. Sci. Budapest. Eötvös Sect. Math., 2 (1959), 25-26.

[6] A. W. Roberts, and D. E. Varberg: Convex functions, Academic Press, New York and London, 1973, 300 pages.

[7] M. R. Tasković: Theory of transversal point, spaces and forks, Monographs of a new theory, Beograd 2003, 1000 pages, to appear.

[8] M. R. Tasković: Nonlinear Functional Analysis, Second Book: Monographs Global Convex Analysis - General convexity, Variational methods and Optimization, Zavod za udžbenike i nastavna sredstva and Vojnoizdavački zavod, Beograd 2001, (in Serbian), 1223 pages.

[9] M. R. Tasković: Transversal spaces, Mathematica Moravica, 2 (1998), 133-142.

Matematički fakultet 11000 Beograd, P.O. Box 550

Serbia \& Montenegro

Received January 7, 2002. 